\title{
Causal Boundary Entropy From Horizon Conformal Field Theory
}

\author{
Ram Brustein \\ Department of Physics, Ben-Gurion University, Beer-Sheva 84105, Israel \\ email: ramyb@bgumail.bgu.ac.il
}

\begin{abstract}
The quantum theory of near horizon regions of spacetimes with classical spatially flat, homogeneous and isotropic Friedman-Robertson-Walker geometry can be approximately described by a two dimensional conformal field theory. The central charge of this theory and expectation value of its Hamiltonian are both proportional to the horizon area in units of Newton's constant. The statistical entropy of horizon states, which can be calculated using two dimensional state counting methods, is proportional to the horizon area and depends on a numerical constant of order unity which is determined by Planck scale physics. This constant can be fixed such that the entropy is equal to a quarter of the horizon area in units of Newton's constant, in agreement with thermodynamic considerations.
\end{abstract}

Typeset using REVTEX 
Black holes possess geometric entropy equal to a quarter of the area of their horizon in units of Newton's constant, known as the Bekenstein-Hawking entropy [1],2]. Since the discovery of black hole entropy, many attempts were made to identify its microscopic, statistical mechanics origin. Strominger [3], and more recently Carlip [四] have argued that the statistical origin of black hole entropy is the ensemble of states of a conformal field theory (CFT) describing fluctuations of two dimensional (2D) horizon surfaces. They have used $2 \mathrm{D}$ methods to evaluate the density of states of this theory, showing that their entropy is indeed a quarter of the horizon area. Some attempts were made to identify the horizon CFT [5], and to extend the results to cosmological de Sitter space [6].

It is widely accepted that geometric entropy must also be attributed to the horizon of de Sitter space [7]. The argument is that de Sitter horizons are event horizons (as are black hole horizons), and therefore a thermodynamic system crossing them is forever removed from an observer's ken. Therefore the loss of the system's entropy must be compensated by an increase of geometric entropy in order for the second law to remain valid. In general, a cosmological horizon is not an event horizon, and a system crossing it is not necessarily forever out of view and so, it may be argued, there is no compelling reason to associate an entropy with a cosmological horizon.

In [8], I have proposed that geometric entropy has to be attributed to cosmological horizons (or, in general, to causal boundaries), whether or not they are event horizons. I have argued that entropy of quantum fluctuations can be lost if the scale of causal connection becomes smaller than their wavelength, for example, in an inflating universe. This is in violation of the second law. The role of proposed geometric entropy is precisely to restore validity of the second law in such situations.

Here I show that the effective quantum theory of near horizon (NH) regions of spacetimes with classical spatially flat Friedman-Robertson-Walker (FRW) geometry is a 2D CFT, appearing due to huge redshifts suffered by horizon fluctuations which allow only massless fluctuations to survive. The central charge of this CFT $c=\alpha \frac{A^{H}}{4 \pi G_{N}}$, is proportional to the horizon area $A^{H}$ in units of Newton's constant $G_{N}$, and depends on a numerical constant 
of order unity $\alpha$, which is determined by Planck scale physics. The expectation value of the Hamiltonian of the theory $L_{0}=\frac{A^{H}}{8 \pi G_{N}}$, is also proportional to the horizon's area in units of Newton's constant. The asymptotic density of horizon states, and therefore the horizon entropy $S^{H}$, can be obtained using Cardy's formula [9] $S^{H}=2 \pi \sqrt{\frac{c}{6}\left(L_{0}-\frac{c}{24}\right)}$ (see [四] for detailed considerations about application of Cardy's formula in this context). The cutoff dependent numerical coefficient $\alpha$ can be set to $\alpha=6$, such that $S^{H}=\frac{A^{H}}{4 G_{N}}$, in agreement with thermodynamic considerations.

Horizons of FRW spaces are not necessarily event horizons, so our results indicate that it is the existence of causal boundary which is the source of geometric entropy, and that for a causal boundary to have geometric entropy it is not required to hold information forever. Our results strongly support the conjecture that causal boundaries and not only event horizons have geometric entropies proportional to their area, and therefore strengthen considerably the conclusion based on this conjecture that a certain class of singularities are thermodynamically forbidden [8] (see also [10]).

Our starting point is the 4D Einstein-Hilbert action

$$
S^{(4)}=\frac{1}{16 \pi G_{N}} \int d^{4} x \sqrt{-g} R^{(4)}+S_{m},
$$

$G_{N}$ being Newton's constant, $g$ the determinant of the $4 \mathrm{D}$ metric $g_{\mu \nu}, R^{(4)}$ is the $4 \mathrm{D}$ Ricci scalar, and $S_{m}$ is the matter action. We consider spatially flat FRW solutions $d s_{4}^{2}=-d t^{2}+$ $a^{2}(t) d r^{2}+a^{2}(t) r^{2}\left(d \theta^{2}+\sin ^{2} \theta d \varphi^{2}\right)$, with expanding scale factors $a(t)=a\left(t_{0}\right)\left(\frac{t}{t_{0}}\right)^{\beta}$. The matter has an ideal fluid type energy momentum tensor derived from $S_{m}$, given by $T_{\nu}^{\mu}=$ $\operatorname{diag}(\rho,-p,-p,-p)$. The energy density $\rho$, and pressure $p$ are related by a simple equation of state $p=w \rho$, which determines the scale factor expansion rate $\beta=\frac{2}{3(1+w)}$. de Sitter space, for which the scale factor expands exponentially, should be considered as the limiting case $w \rightarrow-1, \beta \rightarrow \infty$.

FRW spaces have finite causal connection scale $R_{C C}$ [1], generically called "horizon". Beyond the horizon local interactions are not effective. This causal connection scale is determined by the Hubble parameter $H=\frac{\dot{a}}{a}$, and its derivative: $R_{C C}^{-1}=$ 
$\sqrt{\operatorname{Max}\left[\dot{H}+2 H^{2},-\dot{H}\right]}$. In [11, a covariant expression for $R_{C C}$ is given, but we will approximate it here simply by $R_{C C}=H^{-1}$, a form applicable in almost all situations. For $w<-\frac{1}{3}$ the expansion is inflationary, so fixed comoving points "inside the horizon", will in time "exit the horizon", while for $w>-\frac{1}{3}$ the expansion is decelerated, so fixed comoving points "outside the horizon", will in time "enter the horizon".

Horizons are 2D surfaces that are classically, in the homogeneous and isotropic cases that we are interested in here, well defined spherical shells, which may evolve in time. Quantum mechanically they can fluctuate, so it is reasonable to expect that they can be described effectively by $2 \mathrm{D}$ field theories.

We focus on the $\mathrm{NH}$ geometry by choosing a $2 \mathrm{D}$ metric $d s_{2}^{2}=-d t^{2}+a^{2}(t) d r^{2}$, fixing the position of the horizon $H^{-1}(t)$, at some specified time $t^{*}, H^{-1}\left(t^{*}\right)=d$, and changing coordinates to Schwartzschild-like coordinates $R=a(t) r$, and $T$ defined by $d T=d t+$ $\frac{R / d}{1-R / d} d R$. In the new coordinates

$$
\begin{aligned}
& d s_{2}^{2}=-\left(1-H^{2} R^{2}\right) d T^{2}-2\left[H R-\frac{R}{d} \frac{1-H^{2} R^{2}}{1-\left(\frac{R}{d}\right)^{2}}\right] d R d T \\
& +\left[1-\left(\frac{R}{d}\right)^{2} \frac{1-H^{2} R^{2}}{\left(1-\left(\frac{R}{d}\right)^{2}\right)^{2}}+2 H R \frac{R / d}{1-\left(\frac{R}{d}\right)^{2}}\right] d R^{2} .
\end{aligned}
$$

In the NH "shell" defined by

$$
\frac{H^{-1}(t)-d}{d} \ll \frac{R-d}{d} \ll 1
$$

(2) reduces to

$$
d s_{2}^{2} \simeq-2(1-R / d) d T^{2}+\frac{1}{2} \frac{1}{1-R / d} d R^{2}
$$

One more change of coordinates $d \rho=\frac{1}{2} \frac{1}{1-R / d} d R$, brings (四) into $d s_{2}^{2}=e^{-2 \rho / d}\left(-d T^{2}+d \rho^{2}\right)$ in the NH region $\rho / d \gg 1$, so that $\gamma_{a b}=e^{-2 \rho / d} \eta_{a b}, \sqrt{-\gamma}=e^{-2 \rho / d}$, and $\gamma^{a b}=e^{+2 \rho / d} \eta_{a b}$. For later reference, we note that $t$ is a $\mathrm{NH}$ lightcone coordinate,

$$
t=T-\rho .
$$


We would like to obtain an effective 2D field theory of $\mathrm{NH}$ geometries, so we parametrize the angular part of the metric with a field $\Phi$, which will eventually determine the horizon surface $\langle\Phi\rangle=H^{-1}(t), d s_{4}^{2}=\gamma_{a b}(t, r) d x^{a} d x^{b}+\Phi^{2}(t, r, \theta, \phi)\left(d \theta^{2}+\sin ^{2} \theta d \varphi^{2}\right) . \Phi$ is allowed to have general dependence on the coordinates. For this metric (11) reduces to

$$
S^{(4)}=\frac{1}{8 \pi G_{N}} \int d t d r d \Omega_{2} \sqrt{-\gamma}\left\{\gamma^{a b} \nabla_{a} \Phi \nabla_{b} \Phi+\frac{1}{2} \Phi^{2} R^{(2)}+1\right\},
$$

where $d \Omega_{2}=\sin \theta d \theta d \phi$. In (6) we have dropped the matter action $S_{m}$, since its only function is to determine the classical solution. Note that terms containing angular derivatives are absent in (6) [12].

Mass terms in action (6) are suppressed in the NH region (3) [5]. Obviously, the last term in (6) is exponentially suppressed by a factor $e^{-2 \rho / d}$ with respect to the first two terms! The NH effective action is therefore the following,

$$
S_{\mathrm{NH}}^{(4)}=\frac{1}{8 \pi G_{N}} \int d T d \rho d \Omega_{2} \sqrt{-\gamma}\left\{\gamma^{a b} \nabla_{a} \Phi \nabla_{b} \Phi+\frac{1}{2} R^{(2)} \Phi^{2}\right\} .
$$

We proceed to reduce action (7) to 2D. First, we expand $\Phi$ in spherical harmonics,

$$
\Phi(t, r, \theta, \phi)=\sum_{l,|m| \leq l_{\max }}^{l_{\max }} \Phi_{l, m}(t, r) Y_{l}^{m}(\theta, \varphi) .
$$

The maximal angular momentum $l_{\max }$ in expansion (8) is determined by the short distance cutoff of the theory, as we discuss later. We then substitute (8) into (7), perform the angular integration using the orthogonality property of $Y_{l}^{m}$ 's, and obtain a dimensionally reduced NH 2D effective action,

$$
\begin{aligned}
& S_{\mathrm{NH}}^{(2)}=\frac{1}{8 \pi G_{N}} \int d T d \rho \sqrt{-\gamma} \times \\
& \left\{\sum_{l,|m| \leq l_{\max }}^{l_{\max }} \gamma^{a b} \nabla_{a} \Phi_{l, m} \nabla_{b} \Phi_{l, m}+\frac{1}{2} R^{(2)} \sum_{l,|m| \leq l_{\max }}^{l_{\max }} \Phi_{l, m}^{2}\right\} .
\end{aligned}
$$

Only a single field $\sum \Phi_{l, m}^{2}$, couples to the $2 \mathrm{D}$ curvature term. This field is simply the area of the horizon shell $A^{H}, A^{H}=\int d \Omega_{2} \Phi^{2}(t, r, \theta, \phi)=\sum_{l,|m| \leq l_{\max }}^{l_{\max }} \Phi_{l, m}(t, r)^{2}$. Here we have

\footnotetext{
${ }^{1}$ Strictly speaking, certain variations coming from this term are suppressed
} 
performed the angular integration using the orthogonality property of $Y_{l}^{m}$ 's. We will be interested in fluctuations of the horizon which keep the area fixed at its (time-dependent) classical value, and therefore we will freeze quantum fluctuations of this mode. Since we are interested in counting states for the case of large $l_{\max }$, projecting out a single mode will not compromise the generality of our results. Freezing the quantum fluctuations of the area has the benefit of simplifying quantization of the NH theory enormously. Furthermore, we do not take into account fluctuations of matter sources, assuming that their only effect is to determine the time dependent expectation value of the horizon.

So, when all is said and done, the remaining $\mathrm{NH}$ action is simply a sum of actions of independent'2 free scalar fields, minimally coupled to 2D gravity,

$$
S_{\mathrm{NH}}^{(2)}=\frac{1}{8 \pi G_{N}} \int d^{2} x \sqrt{-\gamma} \sum_{l,|m| \leq l_{\max }}^{l_{\max }} \gamma^{a b} \nabla_{a} \Phi_{l, m} \nabla_{b} \Phi_{l, m} .
$$

Theory (10) can be quantized in the 2D conformal gauge, using standard DDK arguments 13,14. The conformal anomaly of $\Phi_{l, m}$ 's induces a kinetic term for the $2 \mathrm{D}$ conformal mode, and renormalizes the $2 \mathrm{D}$ action such that the full theory is a CFT, whose total central charge vanishes. But the central charge of the Liouville mode cannot be used to calculate the density of states 15,4. For state counting purposes it counts as a single field.

To determine the horizon's entropy we need to compute the effective central charge $c$, of the NH CFT, and the expectation value of its Hamiltonian $L_{0}$. We first calculate the total effective central charge $c$, which is approximately equal to the sum of individual matter central charges,

$$
c \simeq \sum_{l,|m| \leq l_{\max }}^{l_{\max }} 1 \simeq l_{\max }^{2}
$$

In (11) we have neglected contributions from ghosts, from the Liouville mode, ignored the area constraint, and included redundant contributions from a small number of gauge modes, but since we are interested in the case of large $l_{\max }$, we are justified in doing so.

\footnotetext{
${ }^{2}$ We ignore the single overall constraint on their sum, which effectively removes a single field.
} 
The maximal angular momentum $l_{\max }$, is determined by Planck scale physics. The smallest angular variations $\Delta \varphi_{\min }$ and $\Delta \theta_{\min }$ allowed as fluctuations of a sphere of radius $d$, are determined by the short distance cutoff of the theory $\ell_{U V}$,

$$
\Delta \varphi_{\min }=\ell_{U V} / d, \quad \Delta \theta_{\min }=\ell_{U V} / d
$$

Since $Y_{l}^{m} \sim e^{i m \varphi} e^{i l \theta}$, the smallest angular variations $\Delta \varphi_{\min }$ and $\Delta \theta_{\min }$ determine the maximal angular momentum,

$$
m_{\max }=\frac{C_{m}}{\Delta \varphi_{\min }}, \quad l_{\max }=\frac{C_{l}}{\Delta \theta_{\min }},
$$

where $C_{l}, C_{m}$ are numerical coefficients of order unity. We may use eqs. (12,13]) to estimate the maximal allowed angular momentum $l_{\max }^{2}=C_{l} C_{m} d^{2} / \ell_{U V}^{2}$. Assuming that the short distance cutoff is some numerical factor of order unity $k$, times the Planck length $\ell_{U V}=$ $k \sqrt{G_{N}}$, and denoting $\alpha=\frac{C_{l} C_{m}}{k^{2}}$ we obtain our final expression for the total central charge of the NH theory,

$$
c=\alpha \frac{A^{H}}{4 \pi G_{N}} .
$$

The expectation value of $L_{0}$ is determined by the classical background. Recall that the classical solution is a function of time only $\langle\Phi(r, t, \theta, \varphi)\rangle=H^{-1}(t)$, and that $H^{-1}(t)=d\left(\frac{t}{t^{*}}\right)$. But this means that only the $l=0, m=0$ mode has non-trivial expectation value

$$
\left\langle\Phi_{0,0}\right\rangle=\sqrt{4 \pi} d\left(\frac{t}{t^{*}}\right)
$$

To simplify evaluation of $L_{0}$, we go to $2 \mathrm{D}$ lightcone coordinates in two steps, first setting $\tau=\frac{T}{t^{*}}, \sigma=\frac{\rho}{t^{*}}$, and then setting $x^{ \pm}=\tau \pm \sigma$. Note that according to (5), $x^{-}=\frac{t}{t^{*}}$. We expand

$$
\Phi_{0,0}=q+p x^{-}+\sum_{n \neq 0} \frac{1}{n} \alpha_{n} e^{2 i n \pi \frac{\sigma}{\sigma_{\max }} x^{-}},
$$

where $\sigma_{\max }$ determines the range of the $2 \mathrm{D}$ coordinate $\sigma$. Since $x^{-}=\frac{t}{t^{*}}$, we can compare (16) and (15), and observe that for the classical background only $p$ is non-vanishing $p=$ 
$\sqrt{4 \pi} d$, while all the $\alpha_{n}$ 's and $q$ vanish. Since $L_{0}=\frac{1}{8 \pi G_{N}}\left[p^{2}+\sum_{n \neq 0} \alpha_{n} \alpha_{-n}\right]$, it follows that $L_{0}=p^{2} / 8 \pi G_{N}$, but $p^{2}=A^{H}$, so

$$
L_{0}=\frac{A^{H}}{8 \pi G_{N}} .
$$

In general, CFT's have two sets of independent modes which are either functions of $x^{+}$, or $x^{-}$, but the NH theory has only one set of modes [3] [5]. As we show $\Phi_{l, m}=\Phi_{l, m}\left(x^{-}\right)$, leaving only one Virasoro algebra as symmetry of the NH CFT. Near the horizon, as we have already seen, propagating modes are massless, due to redshift effects. But the same redshift effects allow them to propagate only along outgoing light-like trajectories in the $x^{-}$direction. Near black hole horizons, similar redshift effects allow only ingoing modes to propagate. To see this in more detail, we look at the $x^{+}$derivative

$$
\partial_{+}=\frac{1}{2} \partial_{T \mid t}+\frac{1}{2} \partial_{\rho \mid t}=\partial_{T \mid t}=\partial_{\rho \mid t}
$$

where the last equation is obtained using eq.(5). For simplicity, we now set $t^{*}=1$, so $x^{-}=t$. Expressing $T$ and $\rho$ derivatives in terms of $R$ derivatives, we find

$$
\partial_{\rho \mid t} \simeq \partial_{T \mid t} \simeq 2\left(1-\frac{R}{d}\right) \partial_{R}
$$

so smooth functions in the original variables $(R, t)$ have vanishing $x^{+}$derivative in the $\mathrm{NH}$ region (3). Further examination shows that all $x^{+}$derivatives $\partial_{+}^{n}$, vanish for such functions, so smooth functions in the original variables $(R, t)$ are not functions of $x^{+}$but only functions of $x^{-}$, as claimed

We are now ready to use Cardy's formula [9,4] to evaluate the entropy of the horizon,

$$
\begin{aligned}
S_{\text {horizon }} & =2 \pi \sqrt{\frac{c}{6}\left(L_{0}-\frac{c}{24}\right)} \\
& =\frac{A^{H}}{4 G_{N}} \sqrt{\frac{\alpha}{3}\left(1-\frac{\alpha}{12}\right)},
\end{aligned}
$$

\footnotetext{
${ }^{3}$ We will not discuss possible diffeomorphisms anomalies due to the chiral nature of NH theory.
} 
where $\alpha$ is defined above (14).

Since coefficient $\alpha$ is determined by short distance (Planck scale) physics, we expect it to be universal. It should not be sensitive to the macroscopic, large scale physics which determines the exact nature of classical solutions. If so, we may use the limiting case of de Sitter space to "calibrate" it. In de Sitter space the horizon entropy is known to be $\frac{A^{H}}{4 G_{N}}$ from other considerations. This procedure sets the value of $\alpha$ at $\alpha=6$, leading to the conclusion that, in general,

$$
S_{\text {horizon }}=\frac{A^{H}}{4 G_{N}} \text {. }
$$

The horizon shell, whose entropy we have just calculated, is a thin shell, since its thickness is much smaller than its radius, but its thickness is macroscopic, much larger than the Planck length, so we might have expected that its entropy turns out to be proportional to its volume, in units of Planck volume. The entropy of an arbitrary shell does scale as its volume, but not the NH shell. So what is so special about the horizon? Huge redshifts "squash" NH shells, and make their entropy proportional to their area and not to their volume.

Our results apply also to collapsing pressureless matter, since the such systems can be described by FRW metric. In this case, the horizon whose entropy we have computed is an apparent horizon. The apparent horizon reaches the event horizon when all matter has collapsed to a point.

Our explicit calculations were carried out for 4-dimensional, homogeneous and isotropic, spatially flat geometries. But the essential ingredient in our calculation was the huge redshift near the horizon. This left only massless outgoing 2D modes, which are naturally described by a CFT. Since this ingredient seems to be present whenever causal boundaries form, I believe that similar methods can be applied to higher dimensional spaces, along the lines of [4 [6], to more general spatially curved spaces, using the covariant definition of causal connection scale [11], and to string theory along the lines of [16]. 


\section{ACKNOWLEDGMENTS}

I wish to thank S. de Alwis, S. Elitzur, S. Foffa and S. Solodukhin for discussions, and CERN-TH division, where part of this work was carried out, for hospitality. 


\section{REFERENCES}

[1] J. D. Bekenstein, Phys. Rev. D7, 2333 (1973).

[2] S. W. Hawking, Nature 248, 30 (1974).

[3] A. Strominger, JHEP 9802, 009 (1998).

[4] S. Carlip, Phys. Rev. Lett. 82, 2828 (1999); Class. Quant. Grav. 16, 3327 (1999).

[5] S. N. Solodukhin, Phys. Lett. B454, 213 (1999).

[6] F. Lin and Y. Wu, Phys. Lett. B453, 222 (1999).

[7] G. W. Gibbons and S. W. Hawking, Phys. Rev. D15, 2752 (1977).

[8] R. Brustein, Phys. Rev. Lett. 84 (2000) 2072.

[9] J. L. Cardy, Nucl. Phys. B270, 186 (1986); H. W. Bloete, J. L. Cardy and M. P. Nightingale, Phys. Rev. Lett. 56, 742 (1986).

[10] J. D. Bekenstein, Int. J. Theor. Phys. 28, 967 (1989).

[11] R. Brustein and G. Veneziano, hep-th/9912055.

[12] J. H. Yoon, gr-qc/9611050.

[13] J. Distler and H. Kawai, Nucl. Phys. B321, 509 (1989); F. David, Mod. Phys. Lett. A3, 1651 (1988).

[14] J. Polchinski, Nucl. Phys. B324, 123 (1989).

[15] D. Kutasov and N. Seiberg, Nucl. Phys. B358, 600 (1991).

[16] R. Brustein, S. Foffa and R. Sturani, Phys. Lett. B471 (2000) 352. 